\title{
Control PID basado en eventos periódico para sistemas de control en red
}

\author{
E. Aranda-Escolástico ${ }^{1}$, C. Rodríguez ${ }^{1}$, M. Guinaldo ${ }^{1}$, J.L. Guzmán ${ }^{2}$, S. Dormido ${ }^{1}$ \\ ${ }^{1}$ Departamento de Informática y Automática, UNED, España, earandae@bec.uned.es \\ ${ }^{2}$ Departamento de Informática, Universidad de Almería, ceiA3, CIESOL, España
}

\begin{abstract}
Resumen
Este trabajo trata el control basado en eventos en sistemas en red. Se establece un formalismo de control basado en eventos periódico con controladores lineales como el PID a partir del teorema de Lyapunov-Krasovskii para sistemas muestreados. El formalismo permite garantizar la estabilidad asintótica del sistema dado un retardo máximo en la red. Para verificar la efectividad del sistema se ha utilizado el modelo de un reactor inestable con un controlador PI y se ha comparado con un esquema de control periódico.
\end{abstract}

Palabras clave: Control basado en eventos, estabilidad, sistemas en red, retardos.

\section{INTRODUCCIÓN}

Durante las últimas décadas, el constante crecimiento de las tecnologías de comunicación ha atraído con fuerza el interés de la comunidad de control. El uso de redes compartidas para conectar los diferentes elementos de control de forma descentralizada ha supuesto una mejora en términos de mayor flexibilidad de arquitecturas, reducción de los costes de instalación y mantenimiento y una mayor fiabilidad que en las tecnologías de comunicación tradicionales. Paralelamente, este cambio de paradigma lleva asociados una serie de problemas que deben resolverse [12]. Ciertamente, la comunicación a través de una red compartida puede verse afectada por limitaciones en el ancho de banda, retardos o la pérdida de paquetes [9]. Así pues, dado que el ancho de banda disponible es limitado, el muestreo de las diferentes señales puede ser problemático. En algunos protocolos de red, como pueden ser Ethernet o WiFi, el muestreo temporal no es constante, sino que depende fuertemente del tráfico en la red. De esta forma, una elección correcta de los instantes de muestreo puede ayudarnos a maximizar el ancho de banda disponible en nuestro sistema. En este sentido, el control basado en eventos se ha mostrado como una buena alternativa al control periódico ya que ayuda a salvaguardar los recursos de la red $[3,8,10,15,17]$. La idea clave detrás de los sistemas de control basados en eventos es que la información es intercambiada entre las distintas partes del lazo de control únicamente cuando una cierta condición del estado se satisface. Esto es, los instantes de muestreo pasan de estar determinados por el tiempo a estar determinados por el estado del sistema.

Con el mismo objetivo, se han propuesto también otras alternativas como las estrategias de autodisparo (self-triggering; ver $[16,11]$ ). Esta estrategia consiste en realizar una predicción de la evolución del sistema y utilizarla para determinar los instantes de tiempo en los que el lazo de control debe cerrarse. El principal problema de este paradigma es que, dado que los sensores no están monitorizados durante el tiempo entre eventos, pueden ser necesarios tiempos de muestreo muy conservadores con la intención de contrarrestar perturbaciones u otros fenómenos desconocidos. Por el contrario, en el control basado en eventos, se cierra el lazo de control cuando se cumple una condición dependiente del valor actual del estado del sistema, eliminando este problema.

A pesar de estos beneficios, monitorizar el estado de manera continua para detectar los eventos puede ser difícil de conseguir en la práctica cuando trabajamos en una plataforma digital. Además, esto puede implicar un importante gasto de recursos de computación. Una posible alternativa es verificar la condición de disparo solo en instantes de tiempo predefinidos. Esta idea se conoce como control basado en eventos periódico $[7,1]$. Resulta, por tanto, una combinación de las ventajas del control periódico y el control basado en eventos continuo. El sistema se muestrea en instantes discretos de tiempo y la información se transmite únicamente si se considera que es necesario.

Aunque existen múltiples trabajos en la literatura relacionados con diseño y sintonía de controladores basados en eventos, en la mayoría se suele considerar realimentación de estados $[7,18,1]$ y/o monitorización continua de los sensores [2, 5, 14]. Sin embargo, debido a que el controlador PI/PID es el más utilizado en la industria [4], en este artículo se propone un marco para garantizar la estabilidad de en un sistema de control basado en 
eventos periódico con controladores LTI (Linear Time Invariant o Lineal Invariante en el Tiempo) $\mathrm{y}$, por tanto, con un PID. Para ello se utilizan las técnicas desarrolladas para estudiar sistemas con retardo mediante el funcional de LyapunovKrasovskii [6]. Así pues, la principal novedad de este trabajo consiste en establecer un formalismo que permite garantizar la estabilidad asintótica de un sistema en un marco de control PID basado en eventos periódico y bajo limitaciones como retardos variables, propias de comunicaciones a través de la red.

El trabajo se divide de la siguiente forma: En la Sección 2 se plantea el problema y se presenta el marco de control basado en eventos. En la Sección 3 se establece la forma del controlador y se garantiza la estabilidad asintótica del sistema mediante desigualdades matriciales lineales (LMIs). La Sección 4 muestra un ejemplo ilustrativo para probar la efectividad del método propuesto, Finalmente, la Sección 5 recoge las conclusiones del trabajo.

\section{PRELIMINARES}

\subsection{NOTACIÓN}

Sea $x \in \mathbb{R}^{n}$ y $A \in \mathbb{R}^{n \times m}$. Definimos la norma euclidea del vector $x \in \mathbb{R}^{n}$ como $\|x\|:=\sqrt{x^{T} x}$ y $A^{T}$ como la matriz transpuesta de la matriz real $A$. $\quad \lambda_{\max }(A)$ y $\lambda_{\min }(A)$ denotan el máximo y el mínimo autovalor de $A$, respectivamente. Las matrices simétricas $\left(\begin{array}{cc}A & B \\ B^{T} & C\end{array}\right)$ se denotan como $\left(\begin{array}{ll}A & B \\ \star & C\end{array}\right)$.

Además, se denota la matriz simétrica definida positiva $P \in \mathbb{R}^{n \times n}$ como $P>0$, y $P \geq 0, P<0$, y $P \leq 0$ hacen referencia, por tanto, a matrices simétricas semidefinida positiva, definida negativa y semidefinida negativa, respectivamente.

Finalmente, se define el espacio de funciones $\phi:[-h ; 0] \rightarrow \mathbb{R}$, absolutamente continuo en $[-h ; 0]$, y con derivadas de primer orden de cuadrado integrable $W[-h ; 0]$ con norma $\|\phi\|_{W}=$ $\max _{\theta \in[-h, 0]}\|\phi(\theta)\|+\left[\int_{-h}^{0}\|\dot{\phi}(s)\|^{2} d s\right]^{\frac{1}{2}}$. Se denota además $x_{t}(\theta)=x(t+\theta)$, para $\theta \in[-h, 0]$.

\subsection{FORMULACIÓN DEL PROBLEMA}

Considérese el siguiente sistema LTI

$$
\begin{aligned}
\dot{x}(t) & =A x(t)+B u(y(t)) \\
y(t) & =C x(t),
\end{aligned}
$$

donde $x \in \mathbb{R}^{n}$ es el vector de estados, $u \in \mathbb{R}^{m}$ es el vector de entradas, $y \in \mathbb{R}^{p}$ es el vector de salidas y $A, B, C$ son matrices constantes de las dimensiones apropiadas. La condición inicial de (1) viene dada por $x(0)=x_{0}$. En este trabajo, estudiamos un marco de control PID basado en eventos periódico en el que los instantes de transmisión están determinados por un criterio basado en las salidas muestreadas de la planta (obsérvese la Figura 1). Así pues, asumimos que el sistema está conectado a través de una red de comunicación en la que la salida es muestreada periódicamente con periodo de muestreo $h>0$, mientras que los controladores y actuadores son accionados cuando ocurren ciertos eventos. Asumimos también que el retardo de computación en el controlador es despreciable y que la información se transmite en un único paquete y que no hay pérdidas en la red.



Figura 1: Diagrama de bloques. Las señales continuas se representa en línea continua y las señales discretas en línea punteada.

Consideramos en el sistema un retardo variable en el tiempo, $\tau(t)$, debido a la transmisión a través de la red. El retardo debe cumplir $0 \leq \tau_{m} \leq \tau(t) \leq$ $\tau_{M}$, siendo $\tau_{M}$ el máximo retardo admisible.

Puede redefinirse (1), en el esquema descrito, de la siguiente forma:

$$
\begin{aligned}
& \dot{x}(t)=A x(t)+B u\left(y\left(t_{k}\right)\right), \\
& y(l h)=C x(l h),
\end{aligned}
$$

$\forall t \in\left[t_{k}+\tau\left(t_{k}\right), t_{k+1}+\tau\left(t_{k+1}\right)\right)$, siendo $\{l h\}$ con $l \in \mathbb{N}$ el conjunto de instantes de muestreo de la planta tal que $\sup \{l h\} \leq t$ y $\left\{t_{k}\right\}$, con $k \in \mathbb{N}$ un subconjunto de $\{l h\}$, que determina los instantes en los que se ha producido un evento.

Tómese ahora el siguiente error en la salida del sistema:

$$
e(l h)=y\left(t_{k}\right)-y(l h),
$$

entonces $\left\{t_{k}\right\}$ puede expresarse mediante $t_{k+1}=\min \left\{l h>t_{k} \mid f(e(l h), y(l h)) \geq 0\right\}$, donde $f(e(l h), y(l h))$ es la siguiente condición de disparo de eventos:

$$
f(e(l h), y(l h))=\|e(l h)\|-\sigma\|y(l h)\| .
$$


Finalmente, puede definirse $\delta(t)=t-l h$ de forma que

$$
\begin{aligned}
& \dot{x}(t)=A x(t)+B u(y(t-\delta(t))+e(t-\delta(t))), \\
& y(t-\delta(t))=C x(t-\delta(t))),
\end{aligned}
$$

$\forall t \in\left[t_{k}+\tau\left(t_{k}\right), t_{k+1}+\tau\left(t_{k+1}\right)\right)$.

\section{DISEÑO DEL CONTROLADOR}

En esta sección se estudia la estabilidad asintótica del sistema de control basado en eventos periódico (5) bajo la condición de disparo (4). Para ello, haremos uso del siguiente lema:

Lema 1 ([6], Lemma 2). Sean $\alpha_{1}, \alpha_{2} y$ $\alpha_{3}$ números positivos. Si existe un funcional $V\left(t, x_{t}, \dot{x}_{t}\right): \mathbb{R} \times \mathbb{R}^{n} \times \mathbb{R}^{n} \rightarrow \mathbb{R}$ continuo por la derecha para $x(t)$ en (11), absolutamente continuo $y$ diferenciable para $t \neq l$ lh y que satisface

$$
\begin{aligned}
& \epsilon_{1}\|x(t)\|^{2} \leq V\left(t, x_{t}, \dot{x}_{t}\right) \leq \epsilon_{2}\left\|x_{t}\right\|_{W}^{2} \\
& \lim _{t \rightarrow l h^{-}} V\left(t, x_{t}, \dot{x}_{t}\right) \geq\left. V\left(t, x_{t}, \dot{x}_{t}\right)\right|_{t=l h} \\
& \dot{V}\left(t, x_{t}, \dot{x}_{t}\right) \leq-\epsilon_{3}\|x(t)\|^{2}, t \neq l h,
\end{aligned}
$$

entonces el sistema es asintóticamente estable.

En primer lugar, debe establecerse la forma del controlador. De cara al análisis de estabilidad, sin pérdida de generalidad, se asume que la referencia del controlador es cero. La dinámica de un controlador LTI genérico en espacio de estados viene dada por:

$$
\begin{aligned}
\dot{x}_{c}(t) & =A_{c} x_{c}(t)-B_{c} y\left(t_{k}\right) \\
u(t) & =C_{c} x_{c}(t)-D_{c} y\left(t_{k}\right)
\end{aligned}
$$

Utilizando (3), la dinámica del sistema en lazo cerrado resulta

$\dot{x}_{p c}(t)=A_{1} x_{p c}(t)+A_{2} x_{p c}(t-\delta(t))+A_{3} e(t-\delta(t))$,

siendo $x_{p c}^{T}(t)=\left[x(t) x_{c}(t)\right] \mathrm{y}$

$$
\begin{aligned}
A_{1} & =\left(\begin{array}{cc}
A & B C_{c} \\
0 & A_{c}
\end{array}\right) \\
A_{2} & =\left(\begin{array}{cc}
-B D_{c} C & 0 \\
-B_{c} C & 0
\end{array}\right) \\
A_{3} & =\left(\begin{array}{c}
-B D_{c} \\
-B_{c}
\end{array}\right)
\end{aligned}
$$

$\forall t \in\left[t_{k}+\tau\left(t_{k}\right), t_{k+1}+\tau\left(t_{k+1}\right)\right)$.

Puede enunciarse entonces el siguiente teorema:

Teorema 1. Dado un escalar $\sigma>0$, periodo de muestreo $h$, un controlador (9) y bajo la condición de disparo de eventos (4), el sistema (5) es asintóticamente estable si existen matrices $P$, $Q_{1}, Q_{2}, R_{1}, y R_{2}$ con las dimensiones apropiadas, simétricas y definidas positivas tal que

$$
\Pi<0,
$$

con $\Pi$ dado por (13).

$$
\Pi=\left(\begin{array}{ccccc}
\Pi_{11} & \star & \star & \star & \star \\
R_{1} & \Pi_{22} & \star & \star & \star \\
0 & 0 & -Q_{2}-R_{2} & \star & \star \\
\Pi_{41} & R_{2} & R_{2} & \Pi_{44} & \star \\
\Pi_{51} & 0 & 0 & \Pi_{54} & \Pi_{55}
\end{array}\right)
$$

con

$$
\begin{aligned}
\Pi_{11} & =A_{1}^{T} P+P A_{1}+Q_{1}+Q_{2}-R_{1} \\
& +\tau_{m}^{2} A_{1}^{T} R_{1} A_{1}+\bar{\tau}^{2} A_{1}^{T} R_{2} A_{1} \\
\Pi_{22} & =-Q_{1}-R_{1}-R_{2} \\
\Pi_{41} & =A_{2}^{T} P+\tau_{m}^{2} A_{2}^{T} R_{1} A_{1}+\bar{\tau}^{2} A_{2}^{T} R_{2} A_{1} \\
\Pi_{44} & =-2 R_{2}+\Sigma+\tau_{m}^{2} A_{2}^{T} R_{1} A_{2}+\bar{\tau}^{2} A_{2}^{T} R_{2} A_{2} \\
\Pi_{51} & =A_{3}^{T} P+\tau_{m}^{2} A_{3}^{T} R_{1} A_{1}+\bar{\tau}^{2} A_{3}^{T} R_{2} A_{1} \\
\Pi_{54} & =\tau_{m}^{2} A_{3}^{T} R_{1} A_{2}+\bar{\tau}^{2} A_{3}^{T} R_{2} A_{2} \\
\Pi_{55} & =-\mathbb{I}+\tau_{m}^{2} A_{3}^{T} R_{1} A_{3}+\bar{\tau}^{2} A_{3}^{T} R_{2} A_{3}
\end{aligned}
$$

Prueba. Se construye el funcional de Lyapunov como

$$
V(t)=V_{1}+V_{2}+V_{3}
$$

siendo

$$
\begin{aligned}
V_{1} & =x_{p c}^{T}(t) P x_{p c}(t) \\
V_{2} & =\int_{t-\tau_{m}}^{t} x_{p c}^{T}(s) Q_{1} x_{p c}(s) d s \\
& +\int_{t-\bar{\tau}}^{t} x_{p c}^{T}(s) Q_{2} x_{p c}(s) d s \\
V_{3} & =\tau_{m} \int_{t-\tau_{m}}^{t} \int_{s}^{t} \dot{x}_{p c}^{T}(v) R_{1} \dot{x}_{p c}(v) d v d s \\
& +\bar{\tau} \int_{t-\bar{\tau}}^{t-\tau_{m}} \int_{s}^{t} \dot{x}_{p c}^{T}(v) R_{2} \dot{x}_{p c}(v) d v d s
\end{aligned}
$$

donde $P, Q_{1}, Q_{2}, R_{1}$ and $R_{2}$ son matrices simétricas definidas positivas y $\bar{\tau}=\tau_{M}+h$. Tomando la derivada temporal de $V(t)$, se obtiene que

$$
\begin{aligned}
\dot{V}_{1} & =2 x_{p c}^{T}(t) P \dot{x}_{p c}(t) \\
\dot{V}_{2} & =x_{p c}^{T}(t)\left(Q_{1}+Q_{2}\right) x_{p} c(t) \\
& -x_{p c}^{T}\left(t-\tau_{m}\right) Q_{1} x_{p} c\left(t-\tau_{m}\right) \\
& -x_{p c}^{T}(t-\bar{\tau}) Q_{2} x_{p} c(t-\bar{\tau}) \\
\dot{V}_{3} & =\tau_{m}^{2} \dot{x}_{p c}^{T}(t) R_{1} \dot{x}_{p c}(t)+\bar{\tau}^{2} \dot{x}_{p c}^{T}(t) R_{2} \dot{x}_{p c}(t) \\
& -\tau_{m} \int_{t-\tau_{m}}^{t} \dot{x}_{p c}^{T}(s) R_{1} \dot{x}_{p c}(s) d s \\
& -\bar{\tau} \int_{t-\bar{\tau}}^{t-\tau_{m}} \dot{x}_{p c}^{T}(s) R_{2} \dot{x}_{p c}(s) d s .
\end{aligned}
$$




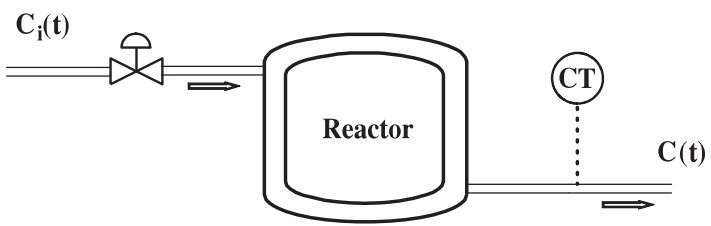

Figura 2: Diagrama del reactor inestable del caso de estudio.

Definimos a continuación el estado extendido

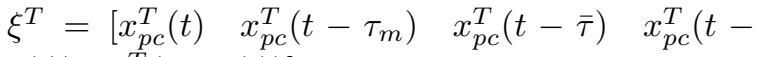
$\left.\delta(t)) \quad e^{T}(t-\delta(t))\right]$ de forma que

$$
\dot{x}_{p c}(t)=\left(\begin{array}{lllll}
A_{1} & 0 & 0 & A_{2} & A_{3}
\end{array}\right) \xi .
$$

Los términos integrales que aparecen en $\dot{V}_{3}$ pueden acotarse mediante la desigualdad de Jensen [1]

$$
\begin{aligned}
& -\tau_{m} \int_{t-\tau_{m}}^{t} \dot{x}_{p c}^{T}(s) R_{1} \dot{x}_{p c}(s) d s \leq \\
& \left(\begin{array}{c}
x_{p c}(t) \\
x_{p c}\left(t-\tau_{m}\right)
\end{array}\right)^{T}\left(\begin{array}{cc}
-R_{1} & R_{1} \\
R_{1} & -R_{1}
\end{array}\right)\left(\begin{array}{c}
x_{p c}(t) \\
x_{p c}\left(t-\tau_{m}\right)
\end{array}\right)
\end{aligned}
$$

y

$$
\begin{aligned}
& -\bar{\tau} \int_{t-\bar{\tau}}^{t-\tau_{m}} \dot{x}_{p c}^{T}(s) R_{2} \dot{x}_{p c}(s) d s \leq \\
& \left(\begin{array}{c}
x_{p c}(t-\delta(t)) \\
x_{p c}(t-\bar{\tau})
\end{array}\right){ }^{T}\left(\begin{array}{cc}
-R_{2} & R_{2} \\
R_{2} & -R_{2}
\end{array}\right)\left(\begin{array}{c}
x_{p c}(t-\delta(t)) \\
x_{p c}(t-\bar{\tau})
\end{array}\right) \\
& +\left(\begin{array}{c}
x_{p c}\left(t-\tau_{m}\right) \\
x_{p c}(t-\delta(t))
\end{array}\right)^{T}\left(\begin{array}{cc}
-R_{2} & R_{2} \\
R_{2} & -R_{2}
\end{array}\right)\left(\begin{array}{c}
x_{p c}\left(t-\tau_{m}\right) \\
x_{p c}(t-\delta(t))
\end{array}\right)
\end{aligned}
$$

Introducimos ahora el término nulo

$0=e^{T}(t-\delta(t)) e(t-\delta(t))-e^{T}(t-\delta(t)) e(t-\delta(t))$,

y acotamos la parte positiva mediante la condición de disparo (4). Finalmente, se obtiene que

$$
\dot{V}(t) \leq \xi^{T} \Pi \xi .
$$

Por consiguiente, queda probado que si se satisfacen las desigualdades (12), entonces el sistema es asintóticamente estable.

\section{RESULTADOS}

En esta Sección se presenta un caso de estudio sobre el que validar el formalismo propuesto y comparar los resultados.
Se ha utilizado como ejemplo un reactor inestable. La dinámica en lazo abierto de algunos reactores químicos puede describirse mediante el modelo de Cholette

$$
\frac{d C(t)}{d t}=\frac{F(t)}{V}\left[C_{i}(t)-C(t)\right]-\frac{k_{1} C(t)}{\left[k_{2} C(t)+1\right]^{2}},
$$

donde $C(t)$ es es la concentración de salida, $C_{i}(t)$ es la concentración de entrada, $F(t)$ es el flujo de entrada, $V=1 \mathrm{~L}$ es el volumen del reactor y $k_{1}=10 \mathrm{Ls}^{-1}, k_{2}=10 \mathrm{mols}^{-1}$. El reactor tiene un punto de operación inestable para $F=0.0333 \mathrm{Ls}^{-1}$ y $C_{i}=3.288 \mathrm{molL}^{-1}$ a $C=1.316 \mathrm{molL}^{-1}$.

Para nuestros propósitos de control, $C_{i}$ es la variable manipulada, $C$ es la variable controlada. Asumismo que existe un retardo mínimo de 1s ya que el transductor necesita cierto tiempo para proporcionar la variable. De la misma forma, asumimos que el retardo máximo, teniendo en cuenta el posible retardo de la red, es de $3 \mathrm{~s}$. El periodo de muestreo de la planta se fija igualmente en $h=3 \mathrm{~s}$.

Linealizando sobre el punto de operación se obtiene que

$$
\begin{aligned}
& A=0.0097 \\
& B=1 \\
& C=0.0333 .
\end{aligned}
$$

El diseño del controlador se ha realizado siguiendo las ideas de [13]. Se ha utilizado por tanto un controlador PI con $T_{i}=43.87$ y $K_{c}=3.29$. Traducido al espacio de estados

$$
\begin{aligned}
& A_{c}=0 \\
& B_{c}=1 \\
& C_{c}=K_{c} T_{i}^{-1}=0.0750 \\
& D_{c}=K_{c}=3.29 .
\end{aligned}
$$

Resolviendo las LMIs del Teorema 1, se obtiene un valor máximo para $\sigma=0.41$. Cuanto más grande sea el valor de $\sigma$, menor será el número de transmisiones pero más se verá afectado el comportamiento de la planta. Se ha simulado el sistema (23) en el caso periódico $(\sigma=0)$ y se ha comparado con $\sigma=0.2$ y $\sigma=0.41$. En la Figura 3 se observa la evolución del estado de la planta mientras que en la Figura 4, se observa la entrada producida por el controlador. Vemos como al aumentar el valor de $\sigma$, el comportamiento se aleja más del caso periódico. Sin embargo, si miramos el número de eventos (Figura 5), las transmisiones se han reducido considerablemente. En el caso periódico se produjeron 100 eventos mientras que en el control basado en eventos periódico se produjeron 46 y 29 eventos para $\sigma=0.2$ y $\sigma=0.41$, 


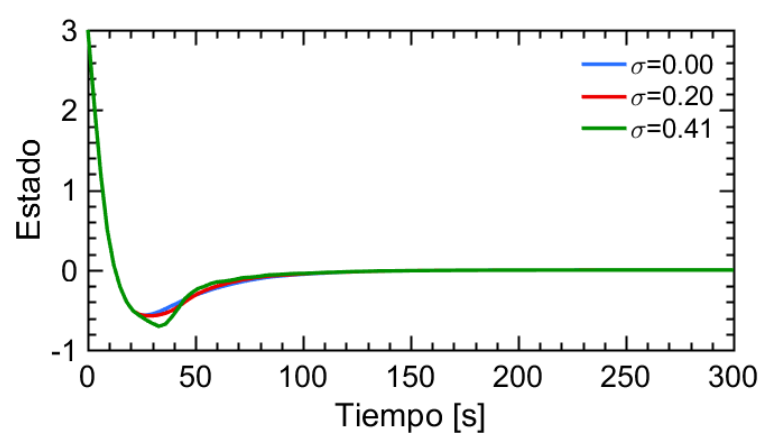

Figura 3: Evolución temporal del estado de la planta.

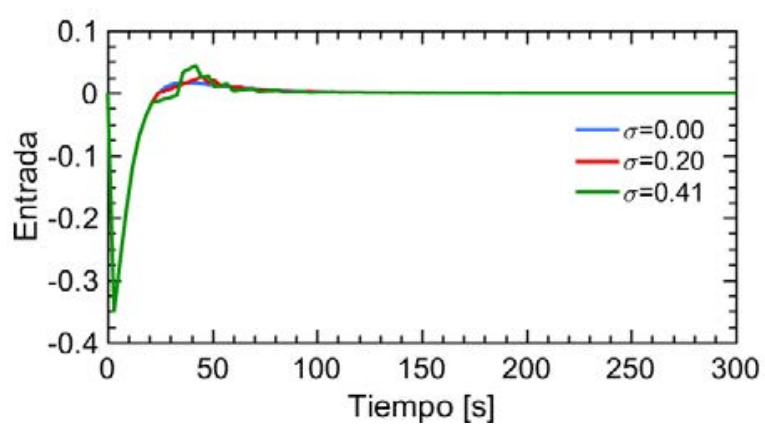

Figura 4: Evolución temporal de la entrada de la planta.

respectivamente. Por otra parte, podemos comprobar otra ventaja del esquema propuesto si empleamos un control basado en eventos continuo, verificando la condición de disparo en todo momento, ya que aparece el conocido efecto Zeno.

\section{CONCLUSIONES}

En este artículo se propone un formalismo de controladores basados en eventos periódicos a partir del teorema de Lyapunov-Krasovskii para sistemas muestreados y con controladores LTI como

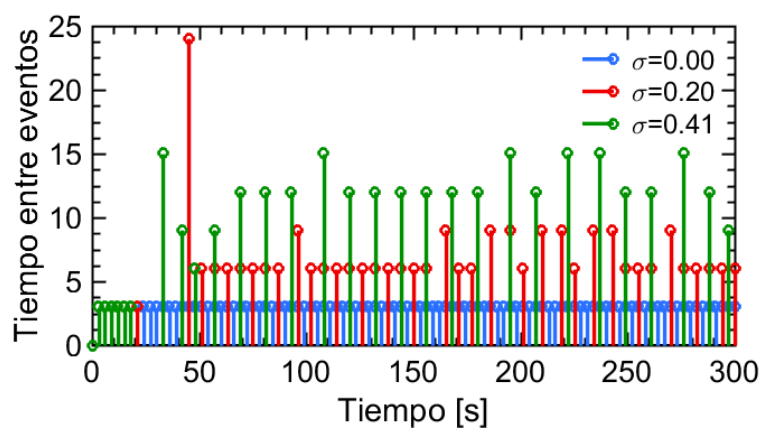

Figura 5: Eventos producidos. La altura de cada marca representa el tiempo desde el anterior evento. es el PID. El formalismo permite estudiar problemas intrínsecos de los sistemas en red como son los retardos variables. Se ha establecido un criterio para garantizar la estabilidad asintótica del sistema a la par que se tiene en cuenta el disparo de eventos para reducir el uso del ancho de banda $y$, por consiguiente, el gasto de recursos de la red.

No obstante, quedan diferentes cuestiones abiertas a estudiar en el futuro. Por un lado, problemas como el rechazo de perturbaciones o pérdida de paquetes pueden estudiarse e incluirse en el formalismo propuesto. Por otra parte, el controlador se asume que ha sido diseñado previamente al análisis de estabilidad. Realizar la síntesis del controlador teniendo en cuenta la propia condición de disparo de eventos puede suponer una mejora en el comportamiento y robustez del sistema.

\section{Agradecimientos}

Este trabajo ha sido financiado en parte por el Plan Promoción de la Investigación de la UNED bajo el proyecto 2014-007-UNED-PROY y por el Ministerio de Economía y Competitividad de España bajo los proyectos DPI2012-31303, DPI2014-55932-C2-1-R y DPI2014-55932-C2-2-R.

\section{Referencias}

[1] E. Aranda-Escolástico, M. Guinaldo, and S. Dormido. A novel approach for periodic event-triggering based on general quadratic functions. In 2015 International Conference on Event-based Control, Communication, and Signal Processing, pages 1-6. IEEE, 2015.

[2] K. E. Årzén. A simple event-based pid controller. In 14th IFAC world congress, 1999.

[3] K. J. Åström and B. M. Bernhardsson. Comparison of periodic and event based sampling for first order stochastic systems. In IFAC Word Conference, pages 301-306, 1999.

[4] K. J. Åström and T. Hägglund. Advanced PID control. ISA-The Instrumentation, Systems, and Automation Society; Research Triangle Park, NC 27709, 2006.

[5] J. Chacón, J. Sánchez, A. Visioli, L. Yebra, and S. Dormido. Characterization of limit cycles for self-regulating and integral processes with pi control and send-on-delta sampling. Journal of Process Control, 23(6):826-838, 2013.

[6] E. Fridman. Introduction to time-delay and sampled-data systems. In European Control Conference, 2014.

[7] W. Heemels, M. Donkers, and A. Teel. Periodic event-triggered control for linear sys- 
tems. IEEE Transactions on Automatic Control, 58(4):847-861, 2013.

[8] W. Heemels, J. Sandee, and P. Van den Bosch. Analysis of event-driven controllers for linear systems. International Journal of Control, 81(4):571-590, 2008.

[9] J. P. Hespanha, P. Naghshtabrizi, and Y. Xu. A survey of recent results in networked control systems. Proceddings IEEE, 95(1):138$162,2007$.

[10] J. Lunze and D. Lehmann. A state-feedback approach to event-based control. Automatica, 46(1):211-215, 2010.

[11] M. Mazo, A. Anta, and P. Tabuada. An iss self-triggered implementation for linear controllers. Automatica, 46(8):1310-1314, 2010.

[12] R. M. Murray, K. J. Åstrom, S. P. Boyd, R. W. Brockett, and G. Stein. Future directions in control in an information-rich world. IEEE Control Systems Magazine, 23(2):20$33,2003$.

[13] J. E. Normey-Rico and E. F. Camacho. Unified approach for robust dead-time compensator design. Journal of Process Control, 19(1):38-47, 2009.

[14] J. Sánchez, A. Visioli, and S. Dormido. A two-degree-of-freedom pi controller based on events. Journal of Process Control, 21(4):639-651, 2011.

[15] P. Tabuada. Event-triggered real-time scheduling of stabilizing control tasks. IEEE Transactions on Automatic Control, 52(9):1680-1685, 2007.

[16] X. Wang and M. Lemmon. Event design in event-triggered feedback control systems. In IEEE Conference on Decision and Control, 2008.

[17] X. Wang and M. Lemmon. On event design in event-triggered feedback systems. Automatica, 47(10):2319-2322, 2011.

[18] D. Yue, E. Tian, and Q. Han. A delay system method for designing event-triggered controllers of networked control systems. IEEE Transactions on Automatic Control, 58(2):475-481, 2013. 